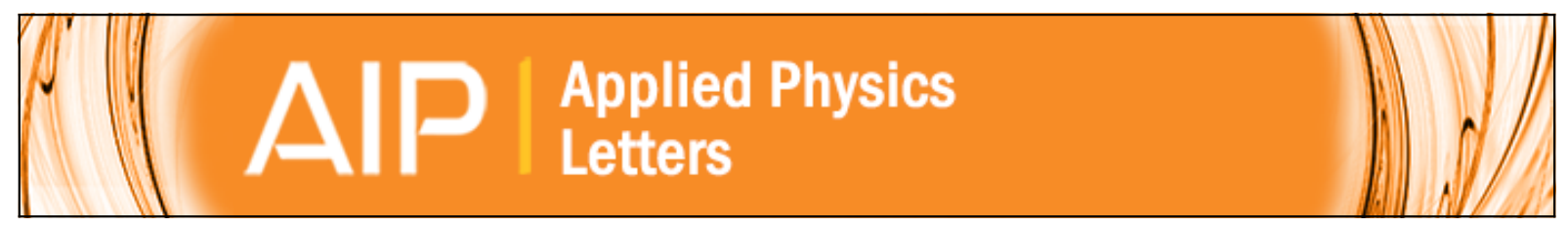

\title{
Role of strain in the blistering of hydrogen-implanted silicon
}

Jung-Kun Lee, Yuan Lin, Q. X. Jia, Tobias Höchbauer, Hyun Suk Jung, Lin Shao, Amit Misra, and Michael Nastasi

Citation: Applied Physics Letters 89, 101901 (2006); doi: 10.1063/1.2345245

View online: http://dx.doi.org/10.1063/1.2345245

View Table of Contents: http://scitation.aip.org/content/aip/journal/apl/89/10?ver=pdfcov

Published by the AIP Publishing

\section{Articles you may be interested in}

Blistering of implanted crystalline silicon by plasma hydrogenation investigated by Raman scattering spectroscopy

J. Appl. Phys. 100, 124906 (2006); 10.1063/1.2401030

Nucleation and growth of platelets in hydrogen-ion-implanted silicon

Appl. Phys. Lett. 86, 154102 (2005); 10.1063/1.1900309

Role of boron for defect evolution in hydrogen-implanted silicon

Appl. Phys. Lett. 83, 3042 (2003); 10.1063/1.1616979

Physical mechanisms behind the ion-cut in hydrogen implanted silicon

J. Appl. Phys. 92, 2335 (2002); 10.1063/1.1494844

Onset of blistering in hydrogen-implanted silicon

Appl. Phys. Lett. 74, 982 (1999); 10.1063/1.123430

\section{NEW! Asylum Research MFP-3D Infinity ${ }^{\text {TM }}$ AFM}

Unmatched Performance, Versatility and Support

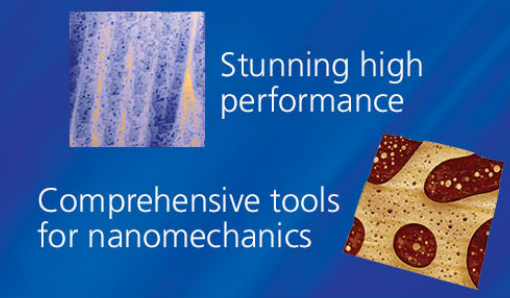

Simpler than ever to GetStarted ${ }^{\mathrm{T}}$

Widest range of accessories

for materials science and bioscience

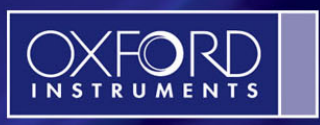

The Business of Science ${ }^{2}$

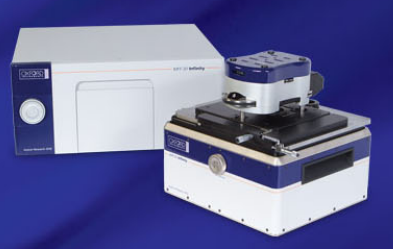




\title{
Role of strain in the blistering of hydrogen-implanted silicon
}

\author{
Jung-Kun Lee, ${ }^{\text {a) }}$ Yuan Lin, Q. X. Jia, Tobias Höchbauer, Hyun Suk Jung, Lin Shao, \\ Amit Misra, and Michael Nastasi \\ Materials Science and Technology Division, Los Alamos National Laboratory, Los Alamos, New Mexico \\ 87545
}

(Received 31 March 2006; accepted 24 July 2006; published online 5 September 2006)

\begin{abstract}
The authors investigated the physical mechanisms underlying blistering in hydrogen-implanted silicon by examining the correlation between implantation induced damage, strain distribution, and vacancy diffusion. Using Rutherford backscattering, scanning electron microscopy, and atomic force microscopy, they found that the depth of blisters coincided with that of maximum implantation damage. A model based on experimental results is presented showing the effect of tensile strain on the local diffusion of vacancies toward the depth of maximum damage, which promotes the nucleation and growth of platelets and ultimately blisters. (C) 2006 American Institute of Physics.
\end{abstract}

[DOI: $10.1063 / 1.2345245]$

The peculiar behavior of hydrogen in Si has stimulated an enormous amount of research over the last few decades. Since hydrogen is mobile and chemically active in $\mathrm{Si}$, hydrogen introduced by ion implantation or plasma hydrogenation interacts easily with both defects and dopants and causes platelets formation and surface blistering. ${ }^{1}$ More recent research has shown that the physical process that leads to the formation of surface blisters in hydrogen implanted silicon can also result in the cleavage of entire layers of silicon, which is being used for integrating high performance electronic components. ${ }^{2}$ While particular attention is paid to the hydrogen induced blistering of Si for its technological importance, the physical nature underlying this process is still evolving. One of the challenging observations under investigation is the role of the method of hydrogen introduction on the crystallographic orientation of platelets. While the platelets in plasma-hydrogenated $\mathrm{Si}$ are parallel to the (111) plane, the platelets formed in hydrogen ion implanted $\mathrm{Si}$ are parallel to the surface of substrate. ${ }^{3-5}$ This difference arouses questions about the dominant driving force for platelets formation and the ultimate blistering or cleaving in $\mathrm{H}$ implanted $\mathrm{Si}$. A recent $a b$ initio study finds that hydrogenated platelets on both (100) and (111) planes have similar free energies leading the authors to conclude that the crystallographic orientation of platelets is determined by kinetic rather than thermodynamic factors. ${ }^{6}$

In the present work, we explore kinetic issues associated with platelet formation in hydrogen-implanted silicon. Implantation damage, hydrogen concentration, and blister morphology and depth were examined for both the roomtemperature and $-140{ }^{\circ} \mathrm{C}$ implanted silicon. Based on our observations, a model is presented linking the effects of tensile strain on the local diffusion of vacancies toward the depth of maximum damage, which will promote the nucleation and growth of platelets and ultimately the formation of blisters.

p-type silicon substrates with $1-10 \Omega \mathrm{cm}$ were ion implanted at room temperature $(\mathrm{RT})$ or $-140^{\circ} \mathrm{C}$ [low temperature (LT)] with $40 \mathrm{keV} \mathrm{H}^{+}$ions at a dose of $5 \times 10^{16} / \mathrm{cm}^{2}$. During implantation, the incident ion beam was oriented $7^{\circ}$ from the surface normal to minimize channeling effects. A

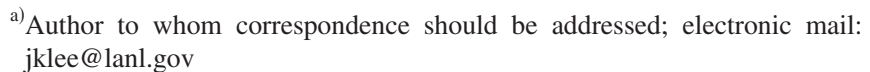

part of the implanted silicon was annealed at $450{ }^{\circ} \mathrm{C}$ for $15 \mathrm{~min}$ to study the platelets formation and blistering behavior.

The lattice damage and the hydrogen concentration in $\mathrm{H}$ implanted samples were analyzed with Rutherford backscattering spectroscopy (RBS) in channeling mode and elastic recoil detection (ERD). The microstructure of annealed samples was examined by using scanning electron microscopy (SEM) and atomic force microscopy (AFM). The strain
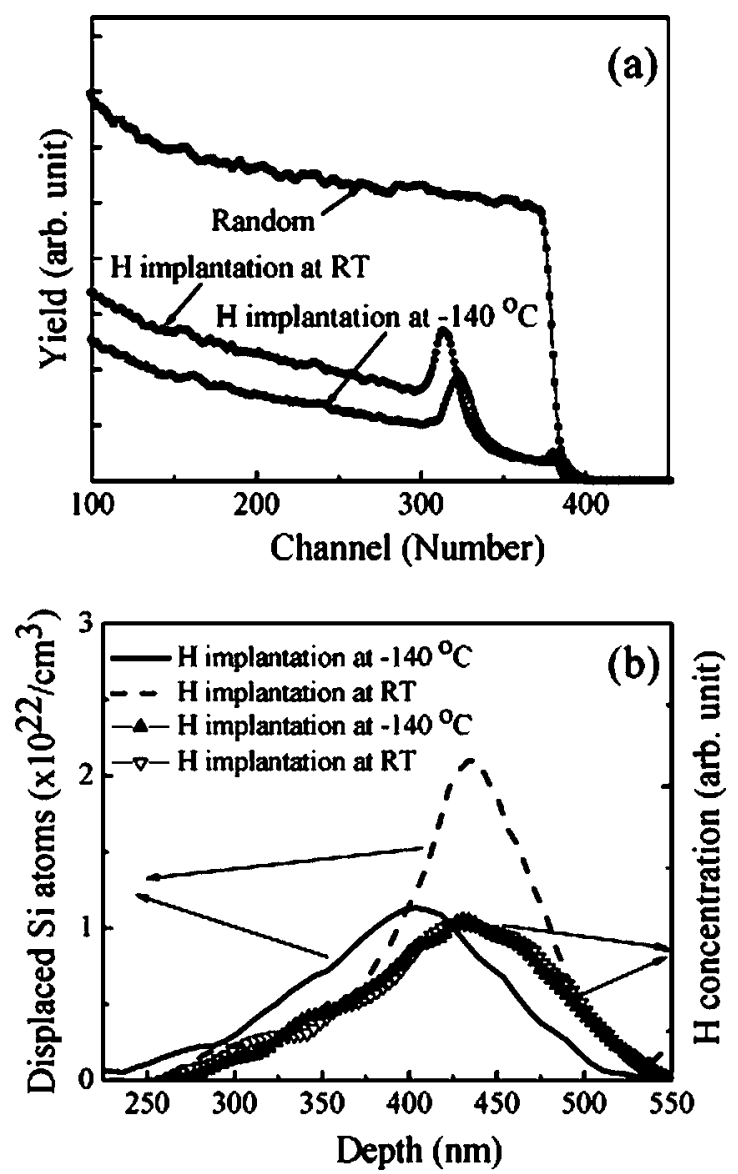

FIG. 1. (a) RBS data in the channeling mode of as-implanted silicon that were hydrogen implanted at $-140{ }^{\circ} \mathrm{C}$ and room temperature. (b) Depth profile of Si displacements deduced from the channeling data of (a) (left axis) and the depth profile of implanted hydrogen deduced from ERD data (right axis). 

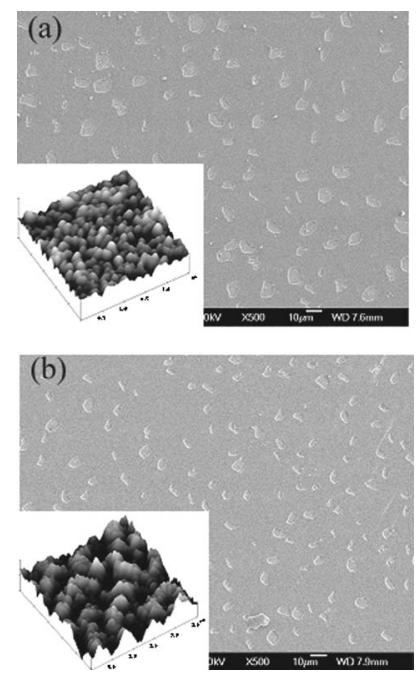

FIG. 2. Scanning electron micrographs of hydrogen implanted Si annealed at $600{ }^{\circ} \mathrm{C}$ for $30 \mathrm{~min}$; (a) for $-140{ }^{\circ} \mathrm{C}$ implanted $\mathrm{Si}$ and (b) for room temperature implanted $\mathrm{Si}$ (inset showing AFM images of blistered region).

depth distribution in the samples after $\mathrm{H}$ implantation was measured by means of high-resolution x-ray diffraction (HRXRD). HRXRD results were autofitted using Bede RADS software to obtain the depth-strain profiles.

Figure 1(a) shows the RBS data in channeling mode for $\mathrm{H}$ implantation at RT and LT, and Fig. 1(b) shows the Si displacement depth distribution profiles deduced from the channeling data presented in Fig. 1(a). The method and the formalism in which these data were obtained are described elsewhere. ${ }^{7}$ These data show that RT implantation provides a deeper and narrower damage distribution. The maximum damage depth for the RT implantation $(430 \mathrm{~nm})$ was deeper than that of the LT implantation $(402 \mathrm{~nm})$ by $28 \mathrm{~nm}$ and the full width at half maximum of the RT implantation damage peak was smaller than that of the LT implantation by $34 \%$. Furthermore, the number of displaced silicon atoms is $46 \%$ higher for the RT implantation compared to the LT implantation. Recently, we have attributed the temperature effect of $\mathrm{H}$ implantation on the damage profiles to in situ chemical interactions between implanted $\mathrm{H}$ and displaced $\mathrm{Si}$ atoms. Essentially, at higher implantation temperatures, irradiation created defects are sufficiently reactive to make a bond with implanted $\mathrm{H}$, thereby stabilizing the defects and reducing defect recombination. Since $\mathrm{H}$ is critical to defect stabilization, the RT damage peak moves towards the maximum in the hydrogen concentration distribution.

The microstructures of LT and RT implanted silicons after annealing at $450{ }^{\circ} \mathrm{C}$ for 15 min were analyzed by SEM and AFM. The blister depths measured by AFM were found to be different for the RT and LT implanted samples. For both cases, the depth of blisters was found to coincide with the depth of maximum damage measured by RBS [Fig. 1(b)]. An average blister depth of $405 \mathrm{~nm}$ was observed for LT implanted Si while RT implanted Si had a blister depth of $434 \mathrm{~nm}$. SEM micrographs and AFM pictures presented in Fig. 2 also show that the blister size and the surface roughness inside the blistered region are strongly correlated with the implantation temperature. The diameter of blisters is larger in RT implanted Si than in LT implanted Si and the root mean square (rms) value of surface roughness is $8.6 \mathrm{~nm}$ for the RT sample and $14.9 \mathrm{~nm}$ for the $\mathrm{LT}$ sample.

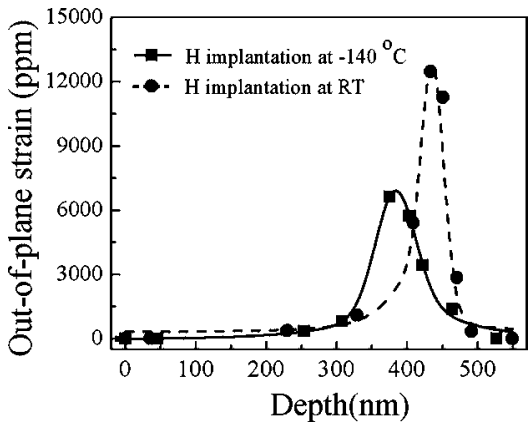

FIG. 3. Strain-depth distributions of as-implanted Si obtained from double crystal x-ray diffraction for $-140{ }^{\circ} \mathrm{C}$ and room temperature implanted $\mathrm{Si}$.

Recently, we have found that the out-of-plane tensile strain present in hydrogen ion implanted Si can bias the diffusivity of vacancies, which in turn influences platelet formation and blistering. ${ }^{8}$ Figure 3 shows the out-of-plane tensile strain profiles of $\mathrm{H}$ implanted $\mathrm{Si}$, measured by double crystal x-ray diffraction. Interestingly, the depths of maximum strain for LT and RT implanted Si are almost identical to their depths of maximum damage. The difference between the LT and RT implantation strain distributions is also similar to the differences observed in their damage distributions The RT sample has a peak strain that is twice, and a strain variance that is about half, compared with the LT sample. The similarities between the strain and damage distributions support the idea that the origin of the out-of-plane tensile strain is due to the presence of defects that are produced by the ion implantation. ${ }^{9,10}$

Since ion implantation generates a nonequilibrium excessive concentration of point defects in $\mathrm{Si}$, these defects are expected to migrate once they become kinetically active during thermal annealing. The flux of vacancies in the presence of a concentration gradient and a strain gradient is described by using two terms. ${ }^{11}$

$$
J=J(\text { concentration })+J(\text { strain }),
$$

where $J$ (concentration) the flux due the gradient in defect concentration and $J$ (strain) represents a flux that results from the gradient in out-of-plane strain. Equation (1) can be rewritten as

$$
\begin{aligned}
J & =-D \frac{d C}{d x}+\frac{D C}{k T} \frac{d E}{d x}, \\
& =-D \frac{d C}{d x}+\frac{D C}{k T} \frac{Y}{1-\nu} V \frac{d \varepsilon}{d x},
\end{aligned}
$$

where $D$ is the diffusion constant, $C$ is the concentration, $k$ is Boltzmann's constant, $T$ is temperature, $E$ is the out-of-plane strain energy, $x$ is the spatial position along the normal direction of Si surface, $Y$ is Young's Modulus, $\nu$ is Poisson's ratio, $\varepsilon$ is the out-of-plane strain, and $V$ is the vacancy volume.

Since the damage profile (Fig. 1) and the strain profile (Fig. 3) are superimposed, two competing vacancy fluxes will exist; given that vacancies can relive local strain, the vacancies diffusion driven by the strain gradient is toward a depth of maximum strain and maximum vacancy concentration, whereas the vacancies diffusion driven by the concentration gradient is away from the depth of the maximum strain and maximum vacancy concentration. If the diffusion 


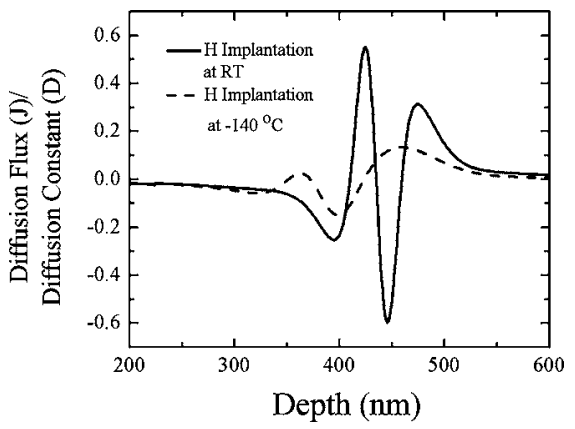

FIG. 4. Vacancy flux calculations of as-implanted silicon, calculated using Eq. (2); (a) $-140{ }^{\circ} \mathrm{C}$ implanted $\mathrm{Si}$ and (b) room temperature implanted Si. A positive flux value indicates diffusion into the bulk and a negative value indicates diffusion towards the surface.

flux driven by the strain gradient is larger than the diffusion flux driven by the concentration gradient, vacancies accumulate at a depth of maximum strain, which will facilitate platelets formation and ultimately blistering. This relationship can be also applied to the interstitials diffusion. However, since the strain at the depth of maximum damage is not relieved by the addition of interstitial atoms but rather is enhanced, the direction of interstitial diffusion driven by the concentration gradient and that of the out-of-plane tensile strain are same.

We calculated the flux of vacancies by using Eq. (3) and the defect and strain profiles in Figs. 1 and 3. In this calculation, the Si vacancy depth distribution was assumed to be identical to the Si displacement depth distribution in Fig. 1. According to the stopping and range of ions in matter (SRIM) simulations, ${ }^{12}$ for light ions such as $\mathrm{H}$ with a relative low energy of $40 \mathrm{keV}$, the distributions for the vacancies and interstitials created by the incident ions are nearly superimposed. The superimposition of vacancy and interstitial distributions justifies the use of RBS channeling data as the $\mathrm{Si}$ vacancy depth distribution. However, this assumption will not be valid for heavy ion or high energy implantation. For heavy ions such as $\mathrm{Ge}$ or $\mathrm{Si}$ and/or incident ion energies on the order of $\mathrm{MeV}$, the interstitial and vacancy distributions would be separated due to the combined effects of dynamic recovery and momentum transfer. ${ }^{13}$ Figure 4 shows the relative total vacancy diffusion flux for RT and LT samples at the beginning of an annealing cycle. Positive flux values correspond to diffusion away from the surface (to greater depths) and negative flux values correspond to diffusion toward the $\mathrm{Si}$ surface. The initial flux calculation shows that the flux of vacancies residing far away from the location of maximum strain is dominated by the concentration gradient and the vacancies tend to diffuse away from the concentration peak. However, in both RT and LT implanted Si, the vacancy flux in the strained region is towards the location of maximum strain. Therefore, the influx of vacancies in the region of high out-of-plane strain exceeds the outflux due to the concentration gradient, resulting in a net influx of vacancies. This indicates that the presence of an out-of-plane tensile strain leads to a supersaturation in vacancies prior to platelet nucleation at a depth of maximum strain, where the hydrogen concentration is also large. Once supersaturated vacancies become kinetically active during thermal annealing, they start to aggregate and form dislocation loops. As supersaturated vacancies are consumed for the nucleation and growth of dislocation loops, the local concentration of free vacancies diminishes and a local vacancy sink is created. Under these conditions the $d C / d x$ term in Eq. (2) will also contribute to the diffusion of vacancies into the region of highest platelet density.

The nucleation energy of vacancy dislocation loops in the presence of an external in-plane stress field can be minimized if the dislocation's Burgers vector is normal to the in-plane stress direction (parallel to out-of-plane strain). ${ }^{8}$ These aligned vacancy dislocations are expected to be easily decorated by the implanted $\mathrm{H}$, since the hydrogenation reaction of vacancies is highly exothermic. We believe that these aligned and hydrogenated dislocations are the in-plane platelets that ultimately lead to the advent of blistering and layer cleaving at the depth of maximum strain. ${ }^{5}$

The vacancy flux description given in Fig. 4 also explains why the blisters of RT implanted Si have smoother surface and larger diameter than those of LT implanted Si. Compared with LT samples, the out-of-plane strain gradient of the RT implanted Si sample is much larger near the depth of maximum strain. Therefore, the driving force for the vacancy flux towards the depth of maximum strain is significantly larger in RT implanted samples (Fig. 4), which results in more effective vacancies aggregation and platelet formation over a narrower region. Under such conditions, platelet growth and ultimate H-bubble formation are highly concentrated and blistering proceeds with minimum zigzag movement that ineffectively consumes the driving force for cleavage and makes the surface of the blistered region rougher. Therefore, blister and cleavage surfaces resulting from hydrogen implantation in silicon will be smoother for a condition that produces steep and narrow out-of-plane strain profiles.

Discussions with John P. Hirth, J. Greg Swadener, and S. S. Lau are gratefully acknowledged. This work has been supported by the U.S. Department of Energy, Office of Basic Energy Sciences and Laboratory Directed Research and Development at LANL.

${ }^{1}$ W. K. Chu, R. H. Kastl, R. F. Lever, S. Mader, and B. J. Masters, Phys. Rev. B 16, 3851 (1977).

${ }^{2}$ M. Bruel, Electron. Lett. 31, 1201 (1995).

${ }^{3}$ G. F. Cerofolini, L. Meda, C. Volpones, G. Ottaviani, J. DeFayette, R. Dierckx, D. Doneli, M. Orlandini, M. Anderle, R. Canteri, C. Claeys, and J. Vanhellemont, Phys. Rev. B 41, 12607 (1990).

${ }^{4}$ T. Höchbauer, A. Misra, M. Nastasi, and J. W. Mayer, J. Appl. Phys. 89, 5980 (2001).

${ }^{5}$ J. K. Lee, M. Nastasi, N. David Theodore, A. Smalley, T. L. Alford, J. W. Mayer, M. Cai, and S. S. Lau, J. Appl. Phys. 96, 280 (2004).

${ }^{6}$ N. Martsinovich, I. S. Martinez, and M. I. Heggie, Phys. Status Solidi C 2, 1771 (2005).

${ }^{7}$ M. Nastasi, T. Höchbauer, R. D. Verda, A. Misra, J.-K. Lee, J. W. Mayer, and S. S. Lau, Nucl. Instrum. Methods Phys. Res. B 219-220, 604 (2004).

${ }^{8}$ M. Nastasi, T. Höchbauer, J.-K. Lee, A. Misra, J. P. Hirth, M. Ridgway, and T. Lafford, Appl. Phys. Lett. 86, 154102 (2005).

${ }^{9}$ S. Milita and M. Servidori, J. Appl. Phys. 79, 8278 (1996).

${ }^{10}$ A. Hedler, S. L. Klaumunzer, and W. Wesch, Nat. Mater. 3, 804 (2004).

${ }^{11}$ P. G. Shewmon, Diffusion in Solids (McGraw-Hill, New York, 1963), p. 24.

${ }^{12}$ J. F. Ziegler, J. P. Biersack, and U. Littmark, The Stopping and Range of Ions in Solids (Pergamon, New York, 1985).

${ }^{13}$ L. Shao, J. Liu, Q. Y. Chen, and W.-K. Chu, Mater. Sci. Eng., R. 42, 65 (2003). 